\title{
Mobile App Support for Electric Vehicle Drivers: A Review of Today's Marketplace and Future Directions
}

\author{
Tai Stillwater, Justin Woodjack, and Michael Nicholas \\ UC Davis PH\&EV Center, 1590 Tilia Street, UC Davis West Village, \\ Davis CA 95616, United States \\ tstillwater@ucdavis.edu
}

\begin{abstract}
Mobile device applications (apps) are becoming an important source of information, control, and motivation for EV drivers. Here we review the current ecosystem of mobile applications that are available for EV drivers and consumers and find that apps are available in six basic categories: purchase decisions, vehicle dashboards, charging availability and payment, smart grid interaction, route planning, and driver competitions. The current range of the EVspecific mobile marketplace extends from pre-sale consumer information, charging information and control, and EV specific navigation features among other services. However, the market is highly fragmented, with applications providing niche information, and using various methodologies. In addition, we find that the barriers to more useful apps are a lack of vehicle and charger APIs (application programming interfaces), lack of data availability, reliability, format and types, and proprietary payment and billing methods. We conclude that mobile applications for EVs are a growing market that provide important direct benefits as well as ancillary services to EV owners, although the lack of uniformity and standards between both vehicle and charger systems is a serious barrier to the broader use of mobile applications for EVs.
\end{abstract}

Keywords: Electric Vehicles, Mobile Apps, Energy Feedback.

\section{Introduction}

Smartphones are becoming more and more common among consumers, making up $54 \%$ of the US mobile subscriber market [1]. This growing ubiquity of connected devices in our population is greatly changing the way consumers both consume and generate information. These mobile devices have near constant internet connection, are usually always with an individual and have many sensors onboard including GPS, accelerometers, cameras, and magnetometers. As governments attempt to increase the adoption of electric vehicles, manufacturer and driver integration of mobile apps will become an increasingly important source of information, control, and motivation. This is because EVs have very different characteristics than conventional vehicles, including most notably their fuel source (electricity compared to gasoline), new units of energy and power (kilowatt-hours and kilowatts instead of gallons of gasoline and horsepower), shorter driving range (typically 50-150 miles), longer refueling times, 
and new refueling locations (home, work, and parking lots). A recent study by the Plug-in Hybrid \& Electric Vehicle (PH\&EV) Research Center found that EV drivers go through a lifestyle learning process to explore and adapt to these vehicle differences over time [2]. The study concluded that consumers' perceptions and use of electric vehicles could be shaped by mobile information technology as it accelerates the lifestyle learning process. This paper attempts to further investigate the current ecosystem of mobile apps tailored for new car buyers and EV drivers and presents some preliminary hypotheses of the possible effects these apps might have on consumer perceptions and behavior.

\section{Current EV Mobile Ecosystem}

The current range of the EV-specific mobile apps extends from pre-vehicle-sale consumer information, charging information and control, EV specific navigation features, and EV-network specific energy efficiency competitions among other services. EV drivers are particularly well suited for mobile apps due to the high adoption rate of smartphones [3]. Figure 1 shows the difference in smartphone adoption between Californian EV drivers and the U.S. general population. As shown in the figure, the most noticeable variation is in the percentage of smartphone adoption rate among EV drivers (84\% compared to $54 \%$ ) and the major difference between iPhone and Android operating systems among those smartphone adopters.

\section{Comparing PEV Drivers and General Population}

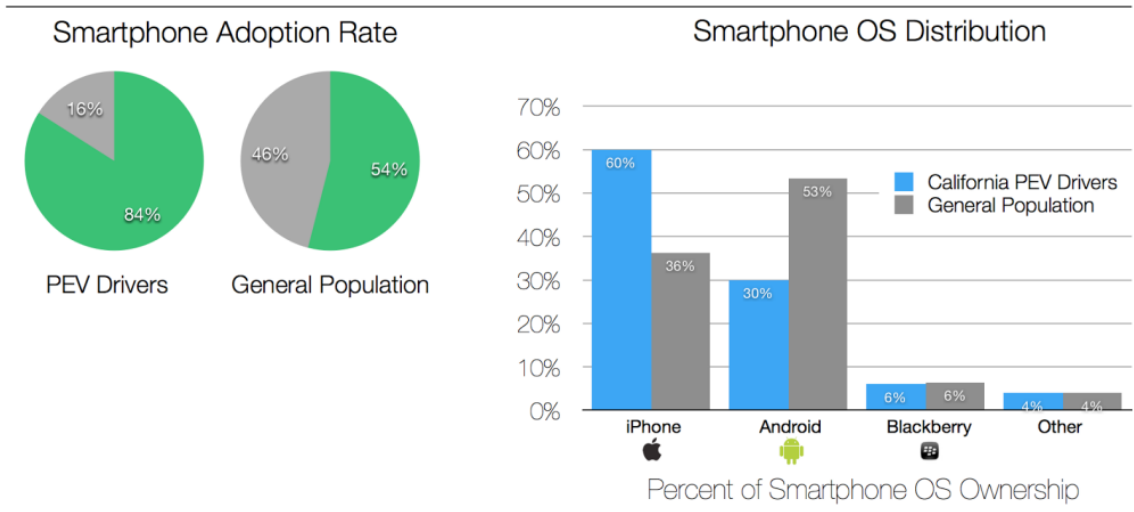

Fig. 1. PH\&EV Research Center survey results comparing California EV drivers with U.S. general population in smartphone adoption rate and the corresponding smartphone operating systems

Clearly there is a high potential for the use of mobile apps to engage, encourage, and even create new EV drivers (by influencing purchase decisions). However, developers face a number of challenges that have limited the capability of mobile apps for EV drivers, including: 
- Lack of vehicle and charger APIs (application programming interfaces)

- Lack of data availability, reliability, format and types

- Proprietary payment and billing methods

Some of these barriers are due to varying levels of manufacturer-specific agreements with software makers and telematics companies. For instance some charging stations have their own RFID (radio-frequency identification) cards that work only within their network, making it more difficult for EV drivers to easily refuel their vehicles in public. Another issue can arise from unknown data quality in apps, such as crowdsourced charging station location and information, potentially reducing the reliability of the information.

Some other problems include the high marginal cost of starting mobile data services. For example, a time-of-use (TOU) charging control app can provide benefits to drivers by leveraging TOU rate tiers to reduce the cost of charging. However, a TOU app that requires a subscription telematics service may not be sensible from a financial perspective.

\section{Mobile App Review}

A search of mobile apps that serve the EV marketplace showed that there are six distinct use cases that have been approached by app developers:

1. Purchase decisions

2. Vehicle dashboards

3. Charging availability and payment

4. Smart grid interaction

5. Route planning

6. Driver competitions

Apps in the purchase decision category provide non-EV drivers with information about EV range, charging availability, or (potential) fuel and emission savings associated with switching to an EV. Mobile technology enables these apps to make use of GPS locations and speed traces to help drivers understand the implications of EV use on their specific route, and with their specific driving patterns.

The vehicle dashboard category covers apps that provide drivers with the ability to check the vehicle battery status remotely, initiate charging, or preheat or cool the vehicle. The remote dashboard typically acts through a cloud service (such as OnStar or Entune) that collects vehicle data on an ongoing basis, and then serves the app with recent information about the vehicle status. This capability, while not unique to EVs, is particularly important for EVs since battery state of charge and charging status can determine if and when a driver can initiate a trip.

The charging availability and payment category is the most active category for developers. These apps provide drivers with maps of charging locations, and may supplement the mapping data with specialty information such as community based comments, photos of chargers, etc. The breadth of choices in this category is due to both the availability of data on charging locations (provided by National Renewable Energy Lab and others), as well as a crowd-sourcing philosophy; created by the early EV driver social networks. Charging payment, however, is currently only served by a 
provider of charging locations. Although this makes intuitive sense, it is clear that integration of a charger reservation or payment system would make many of the other apps much more useful and convenient, and we therefore hope to see developer access to a payment API for privately owned charger networks.

The smart grid integration category is one that is still taking shape as vehicle manufacturers and electric utilities explore the smart grid implications of connected EVs. Very little smart grid interaction is currently possible, so the apps presented in this category are mostly prototypes or demonstrations. The primary use of these apps is to time EV charging to match lower rate tiers or lower environmental impact. Direct smart grid interaction is generally not yet possible because utilities do not release rate, load, or environmental information in a real-time fashion. It is interesting to note that even without demand response infrastructure, utilities could encourage smart grid interaction by simply making rate, load, and carbon intensity data available to developers through an API. This would allow developers to optimize charging schemes based on such utility data.

Route planning is an important aspect of EV driving that is also a complex issue. Currently there are only rudimentary tools available in this category. The route planning category, which we expect to grow in the future as the EV market expands, will cover apps that generate optimal routes, charge points, or charging schemes based on user requested destinations.

Driver competitions are another emerging use of EV connected apps. These tools provide drivers with two primary feedback mechanisms: additional information about driving efficiency and a comparison to peers driving the same model vehicle. These tools can help drivers increase their overall efficiency, and can help automakers engage their customers in a new way. In the future we expect to see competition and efficiency information apps that can allow drivers to compete against affinity groups, cooperate to save fuel, or view savings in comparison to drivers of other vehicles.

Below are the apps identified as of February 2013 for this review.

\subsection{Purchase Decision Apps}

- BMW Evolve: tracks driving patterns to show how much battery power, emissions, and charging cost a drive would consume in a BMW EV

- UC Davis EV Explorer web-app: Allows potential PEV customers to input their travel destinations and frequencies as well as charging infrastructure to find how well PEV technologies can meet their travel needs.

- iEV 2: tracks user trips statistics to help drivers determine if an EV can fulfill a driver's needs, and compares different vehicles

- eMotionApp (Swiss): version of iEV2.

\subsection{Vehicle Dashboard}

- HondaLink EV: an interactive vehicle charging remote control displaying charging status and vehicle range from the mobile app.

- Onstar RemoteLink: mobile app letting you start charging, change charge modes, lock/unlock doors, start volt remotely, search for destinations and send directions to vehicle. Additionally it allows for the viewing of real-time electric range, set 
charge alerts, check current state of charge, view real-time tire pressure information, view latest fuel efficiency figures.

- GreenCharge: app that connects to Nissan Leaf, Plug-in Prius, and Chevy Volt that allows for viewing and sharing of charging history, carbon offset, and historical data to monitor driving habits and average costs to charge.

- Nissan Carwings: app that lets users check state of battery charge, start charging, check when battery charge will complete, see estimated driving range, and remotely turn on or off the climate control system.

- MyFord Mobile: app allows Ford Focus Electric drivers to find chargers and plan trips around charging stations using mapquest. It additional shows the vehicle's state of charge. Additionally it can lock/unlock, remote start of vehicle, precondition the vehicle cabin temperature by setting 'Go Times'.

- Volvo C30 Electric: app for monitoring vehicle including vehicle location, vehicle trip statistics, and owner's manual.

- Better Place Oscar:

- Toyota Entune: an app that houses 'mini-apps' including a fuel economy app, movie and restaurant bookings, sports and stocks information, charge management, remote climate control, vehicle locator, and internet radio.

\subsection{Charging Availability and Charging Payment}

- ChargePoint: app showing the location, real-time status and reservations of chargers within the chargepoint network. It also shows the location of chargers outside its network.

- Blink: allows for finding public Blink chargers and receive charging status updates.

- Recargo:finds public charger from multiple charging networks and crowdsourced locations. Includes social features such as photo sharing and route planning.

- PlugShare: allows drivers to use each-others charge points (home or garage plugs)

- Plugsurfing (europe only): combines public and private charging into one map. Allows plug owners to share their plug on the network.

- CarStations: global database of charging locations. User updated database.

- TipCharge: charger map with fast filtering and routes.

- Chargelocator: includes pay versions with authenticated charger information.

- ChargeYourCar (UK only): is a charge network aggregator and mapping/payment service.

- Onstar: Park-Tap-Charge Prototype app tap phone against charger to select payment options.

\subsection{Smart Grid Interaction}

- MyFord Mobile: enables home charging based on local utility electric rates.

- IBM/EKZ project in Switzerland enables remote charging control and utility-rate dependent charging rules including selection of fossil or renewable energy based on charge timing.

- TENDRIL mobile app: with user selectable charging optimization schemes (fastest, cleanest, cheapest charge). 


\subsection{Route Planning}

- Onstar: Spark EV Waypoint Tab - determines if a waypoint can be reached on a single charge.

\subsection{Driver Competitions}

- Onstar: Volt Driver Challenge App "It will log a Volt's daily and cumulative percentage of electric miles driven, its daily and cumulative miles per gallon, the total gallons of fuel saved, and let drivers compare their numbers to comparable data from other Volt drivers."

- Nissan Carwings: competition mode allows drivers to be ranked based on their driving efficiency, with 4 categories of platinum, gold, silver, bronze 'medals'.

- Toyota Entune: EV ecodriving ranking allows drivers to monitor their ECO status, ECO challenge, and Ranking for reduction of the vehicles carbon footprint.

\section{Discussion and Conclusions}

Mobile apps are transforming the way in which EV drivers interact with their vehicle and the electricity that powers it. Whereas interaction with a gasoline vehicle is much simpler (or at least well known) in terms of the relationship between refueling and driving, the interaction of time, range, and availability of electric "fuel" is both complex and novel. Mobile apps provide information across these categories helping the driver use his or her car in a greater variety of situations and using electricity as fuel more effectively.

This ability to use electricity more effectively has societal implications as well. Being easily able to find a charger in an unfamiliar area reduces the overall requirement to place chargers everywhere. One charger can serve the function of several if they are easy to find and reserve when needed. This reduces overall number of charger installations necessary and lowers the infrastructure barrier needed to encourage wider adoption. Using electricity as a fuel is also beneficial in terms of air quality and greenhouse gas emissions and mobile applications help increase the use of electricity for transportation and adoption of electric vehicles. On average, electricity is less polluting on both metrics.

There are barriers to using these mobile apps and some opportunities for improvement. As presented earlier, mobile apps are at a very early stage of development. There are many single purpose apps, and there is a significant opportunity to integrate functions. Currently there are situations where a user must switch between apps to get all the information he or she needs. For example, charging and vehicle monitoring can be controlled from proprietary apps from auto companies, but there are still other apps such as a charger location app that a user might switch to in order to find the nearest charger. A typical long distance trip may involve locating a charger with one app such as Recargo and finding its network affiliation such as Chargepoint or Blink. Then the user must switch to the app of the network provider to find its availability. Finally while charging, an app from a specific OEM must be used to control 
charging and check state of charge. If vehicle or charger data were available in some secure way, this app switching could be reduced, improving the consumer experience. This presents challenges with data security, but these could be overcome with cooperation between parties.

The same basic data sharing issues prevent EVs from optimizing their charging times based on grid-optimal or price-optimal signals. Currently, utilities do not make such data public or accessible through an API, making it difficult or impossible for 3rd party developers to generate apps that can help optimize charging cost, grid load, or environmental impact.

Mobile applications for EVs are prime for a surge of growth, both as the market penetration of EV grows as well as the integration of currently siloed and fragmented information becomes connected. This mobile app ecosystem could provide important direct benefits as well as ancillary services to EV owners, although the lack of uniformity and standards between both vehicle and charger systems is a serious barrier to the broader use of mobile applications for EVs.

\section{References}

1. ComScore Reports. U.S. Smartphone Subscriber Market Share. comScore, Inc. (December 2012), http: / /www. comscore.com/Insights / Press_Releases / 2013 / 2 / comScore_Reports_December_2012_U.S._Smartphone_Subscriber_Mar ket_Share

2. Woodjack, J., Garas, D., Lentz, A., Turrentine, T.S., Tal, G., Nicholas, M.A.: Consumer Perceptions and Use of Driving Distance of Electric Vehicles: Changes over Time Through Lifestyle Learning Process. Transportation Research Record: Journal of the Transportation Research Board 2287, 1-8 (2012)

3. Tal, G., Nicholas, M., Woodjack, J., Scrivano, D.: Who is Buying Electric Cars in California? Exploring Household and Vehicle Fleet Characteristics of New Plug-in Vehicle Owners 16, UCD-ITS-RR-13-02 (2013) 